\title{
Single isolated attosecond pulse from multicycle lasers
}

\author{
Carlo Altucci, ${ }^{1}$ Rosario Esposito, ${ }^{1}$ Valer Tosa, ${ }^{2}$ and Raffaele Velotta ${ }^{1, *}$ \\ ${ }^{1}$ CNISM-Dipartimento di Scienze Fisiche, Università di Napoli Federico II, via Cintia, 26—Ed. 6-80126, \\ Napoli, Italy \\ ${ }^{2}$ National Institute for R\&D Isotopic and Molecular Technologies, Donath 69-103, 400293 Cluj-Napoca, Romania \\ *Corresponding author: rvelotta@unina.it
}

Received September 4, 2008; accepted October 10, 2008;

posted November 4, 2008 (Doc. ID 101158); published December 5, 2008

\begin{abstract}
We propose a new experimental scheme to produce clean isolated pulses lasting a few hundreds of attoseconds. It is based on high harmonics generation and uses the polarization gating technique combined with the ionization dynamics and the spatial filtering provided by the three-dimensional field propagation. The proposed method is easy to implement, robust against laser parameter fluctuations, and shows to be effective up to a $25 \mathrm{fs}$ pulse duration. (C) 2008 Optical Society of America

OCIS codes: $020.2649,190.4160,320.2250,320.7110$.
\end{abstract}

The availability of a single isolated attosecond pulse (SAP) [1] has paved the way to what has been called attoscience [2]. So far subfemtosecond pulses have been mainly generated by means of high harmonic generation (HHG) in gas, which can be described by the three-step model [3]. In the first step the electron tunnels through the Coulomblike potential barrier that is lowered by the laser electric field, then gains kinetic energy moving in the laser ponderomotive potential, and finally returns to the vicinity of the ionic core, where it recombines and emits an extreme ultraviolet (XUV) photon. It turns out that SAP can be obtained only if one is able to select the HHG emission within half a cycle of the laser field. With fewcycle laser pulses this can be achieved either by selecting spectrally [1] or spatially [4] the harmonics generated in a half cycle by linearly polarized pulses or by using the polarization gating technique, which exploits the fact that a nonlinear elliptical polarization prevents the electron from recolliding $[5,6]$.

A huge breakthrough for obtaining an SAP would be represented by the use of multicycle, rather than few-cycle, laser sources owing to their much higher energy per pulse and possible commercial availability. To this end several schemes have been proposed, although in most cases the required laser pulse is still short ( $<15 \mathrm{fs}$ ) [7] compared to the typical performance of standard laser system ( $>25 \mathrm{fs}$ ). Recently, a polarization gating technique based on the interferometric modulation of the ellipticity of a $50 \mathrm{fs}$ long driving pulse has been put forward [8] leading to a continuum XUV spectrum, which might be possibly associated with SAP. Besides its complexity, this scheme generates the linear polarization gate only in the central part of the driving pulse, thus imposing severe limitations both on the duration and the energy of the driving pulse since medium ionization has to be kept as low as possible before HHG occurs.

In this Letter we propose a new approach to achieve SAP from multicycle pulses delivered by commercial laser systems (Ti:sapphire sources), which combines a polarization gating generated on the leading edge of the driving pulse with a subsequent ion- ization gating $[9,10]$ whose role is to prevent the emission of additional attosecond pulses from the rest of the pulse. Such a method shows to be effective with pulse durations up to $25 \mathrm{fs}$ and can be used with peak intensities as high as $\approx 10^{15} \mathrm{~W} \mathrm{~cm}^{-2}$ or potentially more. A key role in the effectiveness of our method is played by field propagation that acts as a spatial filtering and contributes to the generation of a clean SAP [11,12].

The key idea to produce XUV SAP with multicycle optical pulses is to induce a single half optical cycle (o.c.) time window of nearly linear polarization in the leading edge of the driving pulse. This window must happen at the maximum possible laser intensity for HHG to take place before the significant medium ionization occurs. This is achieved with the superposition of two crossed polarized pulses properly chirped and delayed. A layout of the scheme is illustrated Fig. 1. A linearly polarized input beam is split by a 50-50 beam splitter (BS), which is large enough to allow the recombination in a different area. The large area is required by the need to rotate the polarization of one of the two beams, which is achieved by crossing only once the half-wave plate (HWP) while the phase adjuster (PA) allows one to adjust the relative phase of the two beams.

A robust self-phase modulation (SPM) provides the required chirp (solid curves in Fig. 1) and broadens

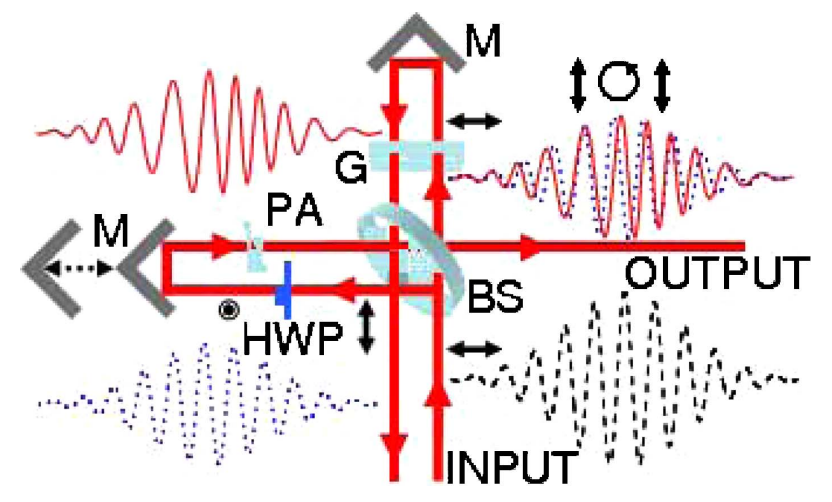

Fig. 1. (Color online) Principle of the experimental scheme. 
the initial spectrum centered at $800 \mathrm{~nm}$ toward the infrared/visible in the leading/trailing edge of the pulse. In view of the role played by the ionization gating only the first half of the pulse needs to preserve its spectral and temporal attributes. The main effect to be concerned about is the group velocity dispersion (GVD) occurring in all the crossed optics, which would lead to pulse lengthening. Since in our method the SAP is generated in the leading edge of the chirped pulse, we can neglect GVD contribution because its importance tends to vanish for wavelengths longer than $800 \mathrm{~nm}$ in most materials (e.g., Ga:La:S glasses). Possible residual positive GVD can be corrected through negative dispersive systems.

Eventually, the output of the interferometer is a superposition of two crossed linearly polarized beams, $E_{x}$ and $E_{y}$, the latter being chirped and delayed,

$$
\begin{aligned}
E_{y}(t, z)= & E_{0 y} \exp \left[-1.39\left(\frac{t-T_{d}}{\tau_{p}}\right)^{2}\right] \sin \left[-\omega_{0}\left(t-T_{d}\right)\right. \\
& \left.+\frac{2 \pi L}{\lambda} n_{2} I\left(t-T_{d}\right)-\zeta(z)+\varphi_{0 y}\right] .
\end{aligned}
$$

In Eq. (1) $E_{0 y}=E_{0} / \sqrt{2}$ with $E_{0}$ being the input electric field amplitude, $T_{d}$ is the relative delay between the output pulses, $\tau_{p}$ is the input pulse duration [FWHM of the intensity $I(t)], \omega_{0}$ is the main oscillation frequency, $L$ and $n_{2}$ are the thickness and the nonlinear refractive index, respectively, of the glass where SPM takes place, $\zeta(z)$ is the Guoy phase, and $\varphi_{0 y}$ is the carrier to envelope phase (CEP). Similarly, $E_{x}$ is described by Eq. (1) without the SPM term with $E_{0 x}=E_{0} / \sqrt{2}$ and setting $T_{d}=0$.

The maximum SPM-induced phase variation experienced by the electric field has been assumed to be 9 rad. It can be easily realized by a laser beam with an intensity of $\approx 50 \mathrm{GW} / \mathrm{cm}^{2}$ crossing a 2 -mm-thick slab of high $n_{2}$ glasses, such as SF59 or Ga:La:S $\left(n_{2}\right.$ $\left.=50-100 \times 10^{-16} \mathrm{~cm}^{2} / \mathrm{W}\right)$. We neglect here a possible spatial variation of the induced SPM owing to radial modulation of the pulse intensity. Such an assumption relies on the possibility to enlarge the beam size and then select only its central part. The value of 9 rad for the SPM-induced phase variation is a tradeoff between a higher value that would generate more linear polarization windows and a lower value that would broaden the single time window. In both cases multiple attosecond pulses would be produced.

The superposition of the two fields given by Eq. (1) leads to an electric field with a linear polarization quite close to the peak intensity, elliptical at maximum intensity, and again linear after the peak. This is shown in detail in Fig. 2(a), where the ellipticity $\varepsilon=E_{y}(t) / E_{x}(t)$ is reported for a laser pulse with $\tau_{p}$ $=20 \mathrm{fs}$. In this simulation the delay between the two pulses is $T_{d}=-0.32 \mathrm{fs}$ (chirped pulse ahead), while the CEP is $0.4 \pi \mathrm{rad}$. The two pulses are in phase $(\varepsilon$ $=0$ ) only for approximately half a cycle, thus realizing the single emission, which is the main requirement for the generation of SAP. This condition takes place

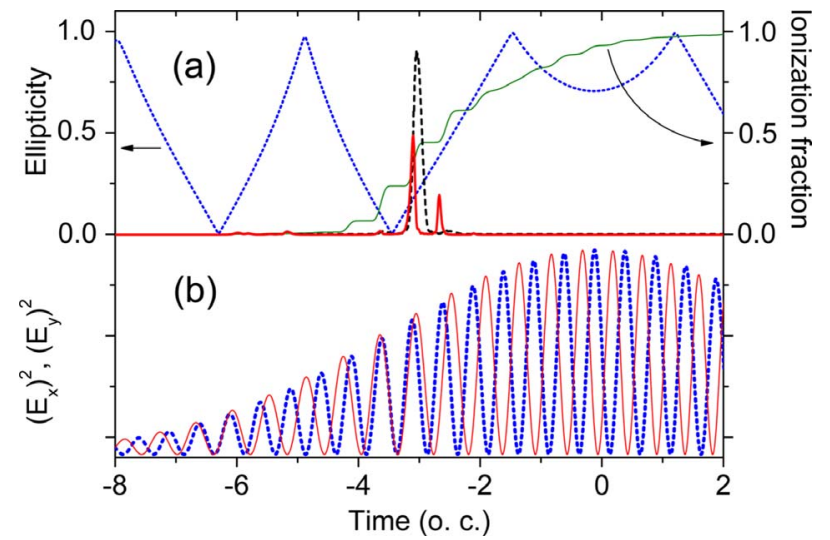

Fig. 2. (Color online) (a) Ellipticity (blue dotted curve), ionization fraction (green thin solid curve), single dipole emission (red thick solid curve), and near-field emission (black dashed curve) versus time for a $\tau_{p}=20 \mathrm{fs}$ pulse. The spectral window is $\mathrm{H} 20-\mathrm{H} 44$ of the fundamental, while other conditions are specified in the text. (b) Chirped $\left(E_{y}\right.$, red solid curve) and chirp-free $\left(E_{x}\right.$, blue dotted curve) squared electric fields.

once in every time window centered at cusp points $\varepsilon=0$, in the leading edge of the laser pulse; however, the recollision events leading to harmonic emission in the spectral interval $\mathrm{H} 20-\mathrm{H} 44$ occur mainly in the time window at $t \approx-3.5$ o.c., since in the former $(t$ $\approx 6.3$ o.c.) the pulse intensity is still too low for an effective HHG. The central part of the pulse is intense enough to fully ionize the medium while the condition $T_{d}=-0.32 \mathrm{fs}$ assures that the ellipticity is quite high $(\approx 0.7)$ at the peak of the combined pulse. The above two factors impede further contributions to the harmonic field from the rest of the pulse. Such a description is supported by the single dipole emission, shown in Fig. 2(a), calculated within the strong field approximation generalized for a field with timedependent ellipticity [13].

Nonadiabatic three-dimensional (3D) propagation of fundamental and harmonic fields through to the gas target has been accounted for by extending the model described in [14] to the case of two fields and by numerically integrating the corresponding propagation equations for both fundamental and harmonic fields. Medium ionization has been calculated by using the nonadiabatic Ammosov-Delone-Krainov model. Throughout our simulations we have assumed a pulse peak intensity at a focus of $8 \times 10^{14} \mathrm{~W} \mathrm{~cm}^{-2}$ and a 1 -mm-long Ar jet of $3.3 \times 10^{3} \mathrm{~Pa}$ local pressure placed at $1.7 \mathrm{~mm}$ in the diverging beam, $3.5 \mathrm{~mm}$ being the confocal parameter.

Our method to generate SAP from multicycle highenergy optical pulses has been found to be very robust against CEP fluctuations. Specifically, in Fig. 3 (a) the total near-field emission is reported as a function of time for three different CEP values showing that a very clean SAP lasting about 380 as is obtained at $t \approx-3.5$ o.c. with $\mathrm{CEP}$ equal to $0.4 \pi \mathrm{rad}$. When CEP shifts by $\pm 0.2 \pi \mathrm{rad}$ an additional satellite [not shown in Fig. 3(a)] appears, having an intensity eight times smaller than the main pulse. The temporal structure degenerates to a double-pulse structure when CEP shifts by more than $\pm 0.3 \pi$ rad from the 

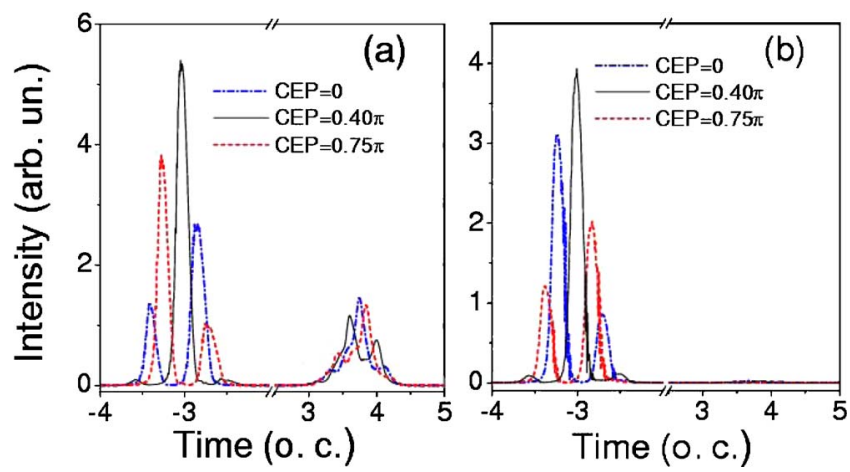

Fig. 3. (Color online) (a) HHG emission in the near field versus time for $\tau_{p}=20 \mathrm{fs}, T_{d}=-0.32 \mathrm{fs}$ (chirped pulse ahead), and $\mathrm{CEP}=0$ rad (blue dotted-dashed curve), CEP $=0.4 \pi \mathrm{rad}$ (black solid curve), and $\mathrm{CEP}=0.75 \pi \mathrm{rad}$ ( $\mathrm{red}$ dashed curve). (b) Corresponding far field emission assuming a $0.7 \mathrm{~mm}$ diameter aperture placed at $0.5 \mathrm{~m}$ from the interaction region.

optimum value. Due to its high divergence, the second emission occurring approximately 7 o.c. later is completely removed in the far field [Fig. 3(b)] by simply inserting an appropriate on-axis aperture. Our simulation reveals that this spurious emission at 7 o.c. after the main one [see Fig. 3(a)] comes from off-axis contributions as a result of an incomplete peripheral depletion of the medium and of an additional time gate of nearly linear polarization occurring in the trailing edge of the pulse. Further analysis of the near field shows that such contributions are characterized by high angular divergence (more than $4 \mathrm{mrad}$ against $1-2 \mathrm{mrad}$ of the main pulse). This is demonstrated by the far field calculation carried out assuming a $0.7 \mathrm{~mm}$ diameter aperture placed at $0.5 \mathrm{~m}$ from the gas-jet and reported in Fig. 3(b); in fact, the aperture, while it only slightly chokes the main pulse, completely cuts the subsequent peak. The SAP generation finds its counterpart in the frequency domain as shown in Fig. 4; the best case, for $\mathrm{CEP}=0.4 \pi \mathrm{rad}$, gives rise to a supercontinuum spec-

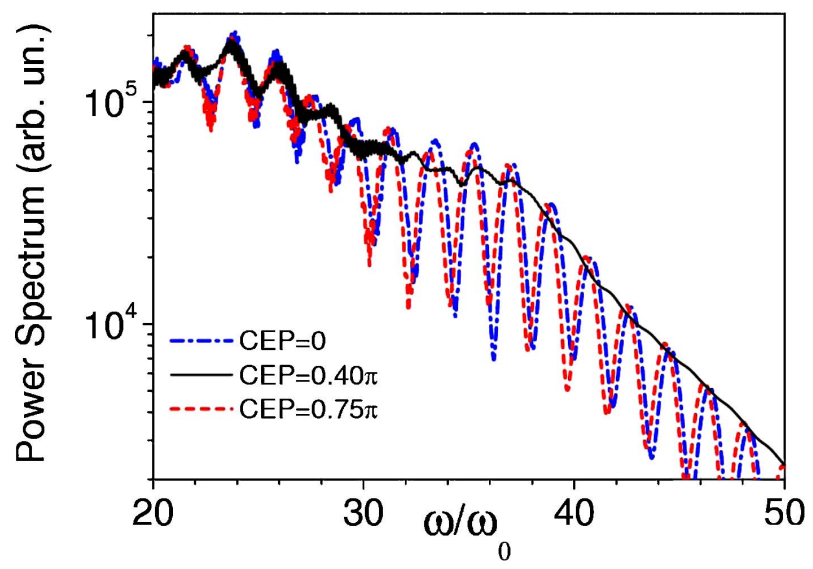

Fig. 4. (Color online) Spectra of the near-field emission reported in Fig. 3(a). trum while strong spectral modulation occurs when the double peak structure arises in time.

Our analysis has also evidenced a robust stability against pulse peak intensity $\left(I_{0}\right)$ and pulse delay $\left(T_{d}\right)$ fluctuations. Indeed, SAP generation with a contrast ratio better than 1:4 still takes place when $\Delta T_{d}$ $\leqslant 0.20 \mathrm{fs}$ and $\Delta I_{0} / I_{0} \leqslant 2.2 \%$. These tolerances are fully achievable in practice with currently available technology. We also checked the performances with $\tau_{p}=25 \mathrm{fs}$ finding SAP with a contrast ratio better than 1:10 in the best conditions.

In conclusion, our method leads to high brightness SAP generated thanks to a novel physical interplay among polarization and ionization gating combined with 3D transient phase matching. The experimental scheme is easy to implement in a typical ultrafast laser facility and therefore represents a crucial step to enable the access to attoscience to a wide scientific community.

This work was partially performed in the framework of the Italia-Romania Agreement on Scientific and Technological Cooperation. V. Tosa acknowledges partial support from contract 372, Romanian Second National Research Plan.

\section{References}

1. R. Kienberger, E. Goulielmakis, M. Uiberacker, A. Baltuska, V. Yakovlev, F. Bammer, A. Scrinzi, T. Westerwalbesloh, U. Kleineberg, U. Heinzmann, M. Drescher, and F. Krausz, Nature 427, 817 (2004).

2. P. B. Corkum and F. Krausz, Nat. Phys. 3, 381 (2007).

3. P. B. Corkum, Phys. Rev. Lett. 71, 1994 (1993).

4. C. A. Haworth, L. E. Chipperfield, J. S. Robinson, P. L. Knight, J. P. Marangos, and J. W. G. Tisch, Nat. Phys. 3, 52 (2007).

5. L. J. Sola, E. Mével, L. Elouga, E. Constant, V. Strelkov, L. Poletto, P. Villoresi, E. Benedetti, J. P. Caumes, S. Stagira, C. Vozzi, G. Sansone, and M. Nisoli, Nat. Phys. 2, 319 (2006).

6. C. Altucci, C. Delfin, L. Roos, M. B. Gaarde, A. L'Huillier, and I. Mercer, Phys. Rev. A 58, 3934 (1998).

7. P. Lan, L. Peixiang, C. Wei, W. Xilin, and W. Hong, Opt. Lett. 32, 1186 (2007).

8. P. Tzallas, E. Skantzakis, C. Kalpouzos, E. P. Benis, G. D. Tsakiris, and D. Charalambidis, Nat. Phys. 3, 846 (2007).

9. H. J. Shin, D. G. Lee, Y. H. Cha, K. H. Hong, and C. H. Nam, Phys. Rev. Lett. 83, 2544 (1999).

10. A. Jullien, T. Pfeifer, M. J. Abel, P. M. Nagel, M. J. Bell, D. M. Neumark, and S. R. Leone, Appl. Phys. B 93, 433 (2008).

11. C. Altucci, V. Tosa, and R. Velotta, Phys. Rev. A 75, 061401 (2007).

12. M. B. Gaarde, J. L. Tate, and K. J. Schafer, J. Phys. B 41, 132001 (2008).

13. P. Antoine, A. L'Huillier, and M. Lewenstein, Phys. Rev. A 53, 1725 (1996).

14. V. Tosa, H. T. Kim, I. J. Kim, and C. H. Nam, Phys. Rev. A 71, 063807 (2005). 